\title{
Analysis on the Effects of Shensong Yangxin Capsules and Amiodarone on Heart Rate Variability Indices and Inflammatory Factors in Patients with Arrhythmia
}

\author{
Yongjie Wang*, Hongxing Zhang, Wangbo Cao \\ Dezhou Hospital of Traditional Chinese Medicine, Dezhou 253013, Shandong Province, China
}

\begin{abstract}
[Abstract] Objective: To study the effects of Shensong Yangxin capsules and amiodarone on heart rate variability indexes and inflammatory factors in the treatment of patients with arrhythmia. Methods: The research subjects were selected patients with arrhythmia admitted in our hospital's department from October 2018 to October 2019, and a total of 70 patients were divided into two groups. 35 subjects in the control group were treated with amiodarone alone, and 35 subjects in the observation group were treated with Shensongyangxin capsule in combination with amiodarone for comparisons in treatment effects, heart rate variability indices, inflammatory factors, and adverse reactions. Results: After observation, the treatment efficiency in the observation group was higher than that in the control group $(\mathrm{P}<0.05)$. Before treatment, there was no difference in heart rate variability indices between the two groups $(\mathrm{P}>0.05)$, after treatment, the improvement in patients of the observation group was better than that of the control group $(\mathrm{P}<0.05)$. After treatment, the inflammatory factors of the two groups of patients decreased, and the indicators of the observation group were comparably lower than those of the control group $(\mathrm{P}<0.05)$. In the comparison of adverse reactions, the observation group showed a lower incidence of adverse reactions after treatment than the control group $(\mathrm{P}<0.05)$. Conclusion: In the treatment of patients with arrhythmia, the combined use of Shensong Yangxin capsules and amiodarone has more significant therapeutic effects, which can effectively improve the patient's heart rate variability indices, reduce the inflammatory factors, and relieve the symptoms in patients. The incidence of adverse reactions is low and the safety is quite high, therefore it has great value in clinical applications.
\end{abstract}

Key words: Shensong Yangxin capsules; Amiodarone; Arrhythmia; Heart rate variability indices; Inflammatory factors

Publication date: May, 2021; Publication online: 31 May, 2021

*Corresponding author: Yongjie Wang, wangyongjiedezhou@163

\section{Foreword}

With the continuous societal development and the increasing aging of population, cardiovascular disease has gradually become a disease that poses a serious threat to people's health. Arrhythmia is quite common in cardiovascular diseases, often observed in patients with organic heart disease. The progress of persistent arrhythmia can cause heart failure and sudden arrest, which is a huge threat to people $^{[1]}$. In the treatment of diseases, drug therapy is a commonly used as a treatment method which can effectively control the patient's condition. However, most of the medicines for diseases are Western medicines. After taking them for a long time, patients had some adverse reactions, which affected the treatment effects. In related research, Shensong Yangxin capsules were found having good therapeutic effects on arrhythmia ${ }^{[2]}$. This study analyzed and explored the effects of Shensong Yangxin capsules and amiodarone on heart rate variability indices and inflammatory factors in patients with arrhythmia. The research report is as follow.

\section{Information and methods}




\subsection{General information}

The research subjects were selected patients with arrhythmia admitted in our hospital's department from October 2018 to October 2019, and a total of 70 patients were selected by blind selection. In the 35 patients control group, 20 were male patients and 15 were female patients, raging in age from 49 to 80 years old, with an observed average of (61.5 \pm 14.6$)$ years old; in the 35 patients observation group, there were 22 male patients and 13 female patients, all in the age range of 50-84 years, and the observed average was $(64.1 \pm 15.2)$ years old. After comparison, the observed information between the two groups of patients was balanced and comparable $(\mathrm{P}>0.05)$.

\subsection{Inclusion and exclusion criteria}

\subsubsection{Inclusion criteria}

(1) Diagnosed as arrhythmia after clinical manifestations, electrocardiogram, ultrasound and other examinations, and no influence from other diseases; (2) The patient's clinical data was complete; (3) The patient had an understanding of the contents of this study and agreed to the study; (4) The research protocol was approved by the hospital ethics committee.

\subsubsection{Exclusion criteria}

(1) Congenital heart disease or severe dysfunction of other vital organs; (2) Suffered from acute trauma or infection recently; (3) Allergic to the drugs used in the study.

\subsection{Methods}

\subsubsection{Control group}

After basic treatment, patients were treated with amiodarone alone. Taken orally 2 times a day, each time $0.2 \mathrm{~g}$. During the treatment period, the dosage is increased or decreased according to the patient's conditions.

\subsubsection{Observation group}

On the basis of the amiodarone treatment in the control group, Shensong Yangxin capsule was added to the treatment. $1.6 \mathrm{~g}$ of the capsule was taken orally each time, 3 times a day. After 1 month of treatment, the two groups of patients were compared and analyzed.

\subsection{Observation indicators}

\subsubsection{Clinical treatment efficiency}

The clinical treatment efficiency of the two groups of patients were compared and divided into significantly effective, effective and ineffective. Total efficiency = incidence proportion of significantly effective + incidence proportion of effective.

\subsubsection{Heart rate variability}

The heart rate variability indices of the two groups of patients before and after treatment were compared, including the mean RR interval standard deviation (SDNN index), the 24hR-R interval standard deviation (SDNN), and the root mean square of the difference between adjacent normal RR intervals Value (rMSSD) and other indicators.

\subsubsection{Changes in inflammatory factors}

The changes in inflammatory factors (hypersensitive C-reactive protein, Hs-CRP, interleukin-6: interleukin-6, IL6) before and after treatment between the two groups were compared.

\subsubsection{Adverse reactions of patients}

The adverse reactions of patients, such as abdominal distension, constipation, dizziness, nausea and other symptoms recorded and analyzed.

\subsection{Treatment efficiency standard}

Diagnostic criteria of clinical symptoms: significantly effective: the patient's clinical symptoms disappeared or have been significantly improved; effective: the patient's clinical symptoms have been relieved or disappeared to a large extent; ineffective: the clinical symptoms has not been significantly improved or the symptoms have increased after treatment.

Diagnostic criteria for ECG treatment efficacy: significantly effective: the patient's premature contraction decreased by $90 \%$ or more, and the symptoms of arrhythmia basically disappeared; effective: the patient's premature contraction decreased by $50 \%$ to $90 \%$, or the number of episodes of arrhythmia symptoms decreased by at least $50 \%$; ineffective: the premature contraction decreased by less than $50 \%$, and the symptoms of arrhythmia were not significantly improved.

\subsection{Statistical analysis}

The test data of this experiment was effectively entered into SPSS22.0, the measurement data is expressed in the form of $(\bar{x} \pm s)$, and the t-test is used for statistical analysis; the count data is expressed in rate and percentage, and the chi-square value test was used for statistical analysis. The test value $\mathrm{P}<0.05$ indicates statistically significant difference.

\section{Results}

\subsection{Comparison of the clinical treatment efficiency between the two groups of patients}

In the comparison of the two groups of patients, the total treatment efficiency of the observation group was $94.29 \%$ 
$(33 / 35)$, and that of the control group was $77.14 \%(27 / 35)$. After observation, the total treatment efficiency in the observation group was higher than that of the control group $(\mathrm{P}<0.05)$. See Table 1.

Table 1. Comparison of the clinical treatment efficiency between the two groups of patients [n (\%) ]

\begin{tabular}{cccccc}
\hline Group & No. of Cases & Significantly Effective & Effective & Ineffective & Total Efficiency \\
\hline Observation & 35 & $18(51.43)$ & $15(42.86)$ & $2(5.71)$ & $33(94.29)$ \\
Control & 35 & $15(42.86)$ & $12(34.29)$ & $8(22.86)$ & $27(77.14)$ \\
$\chi^{2}$ & - & - & - & - & 4.200 \\
P & - & - & - & - & 0.040 \\
\hline
\end{tabular}

\subsection{The heart rate variability indices of the two} groups of patients before and after treatment

Before treatment, there was no difference in the heart rate variability indices of the two groups of patients $(\mathrm{P}>0.05)$.
After treatment, the improvement in the observation group was better than that of the control group $(\mathrm{P}<0.05)$. See Table 2 .

Table 2. Comparison of the heart rate variability indices of the two groups of patients before and after treatment $(\bar{x} \pm s, \mathrm{~ms})$

\begin{tabular}{cccccc}
\hline Time & No. of Cases & Group & SDNN Index & SDNN & rMSSD \\
\hline \multirow{2}{*}{ Before Treatment } & \multirow{2}{*}{35} & Observation & $75.37 \pm 8.31$ & $86.20 \pm 18.23$ & $16.44 \pm 10.19$ \\
t & & Control & $75.46 \pm 8.38$ & $86.23 \pm 18.26$ & $16.66 \pm 10.22$ \\
P & - & - & 0.045 & 0.007 & 0.090 \\
& - & - & 0.964 & 0.995 & 0.928 \\
After Treatment & \multirow{2}{*}{35} & Observation & $96.03 \pm 10.12$ & $116.79 \pm 23.22$ & $30.05 \pm 12.02$ \\
t & - & Control & $85.88 \pm 9.94$ & $99.03 \pm 20.15$ & $21.98 \pm 11.93$ \\
P & - & - & 4.233 & 3.418 & 2.819 \\
\hline
\end{tabular}

\subsection{Comparison of changes in inflammatory factors} between the two groups of patients before and after treatment

Before treatment, there was no difference in the inflammatory factors between the two groups of patients
$(\mathrm{P}>0.05)$. After treatment, the inflammatory factors of the two groups of patients decreased, and the indicators of the observation group were lower than those of the control group, which was comparable $(\mathrm{P}<0.05)$. See Table 3 .

Table 3. Comparison of changes in inflammatory factors between the two groups of patients before and after treatment $(\bar{x} \pm s)$

\begin{tabular}{ccccc}
\hline Time & Cases & Group & Hs-CRP ( mg/L) & IL-6 (pg/L) \\
\hline Before & & Observation & $18.79 \pm 5.21$ & $38.28 \pm 2.12$ \\
Treatment & 35 & Control & $18.97 \pm 5.14$ & $38.39 \pm 2.13$ \\
T & - & - & 0.146 & 0.217 \\
P & - & - & 0.885 & 0.829 \\
After & 35 & Observation & $7.68 \pm 2.21$ & $19.02 \pm 1.97$ \\
Treatment & - & Control & $12.18 \pm 3.49$ & $23.15 \pm 2.16$ \\
T & - & - & 6.445 & 8.358 \\
P & - & - & 0.000 & 0.000 \\
\hline
\end{tabular}

3.4 Comparison on the occurrence of adverse reactions between the two groups of patients
In the comparison of adverse reactions, the incidence of adverse reactions in the observation group after treatment 
was lower than that of the control group $(\mathrm{P}<0.05)$. See Table

4.

Table 4. Comparison on the occurrence of adverse reactions between the two groups of patients [n (\%) ]

\begin{tabular}{cccccccc}
\hline Group & No. of Cases & \multicolumn{1}{c}{ Bloating } & Constipation & Nausea & Dizziness & Total Incidence Rate \\
\hline Observation & 35 & $1(2.86)$ & $1(2.86)$ & $1(2.86)$ & $1(2.86)$ & $4(11.43)$ \\
Control & 35 & $2(5.71)$ & $3(6.67)$ & $4(11.43)$ & $2(5.71)$ & $11(31.43)$ \\
$\chi^{2}$ & - & & - & - & - & - & 4.158 \\
P & - & & - & - & - & 0.041 \\
\hline
\end{tabular}

\section{Discussion}

Amiodarone is a sodium ion channel blocker that can dilate blood vessels, improve myocardial blood supply, and prolong the action potential and effective refractory period of ventricular and atrial muscles. It has significant effects on the treatment of patients with arrhythmia and is regarded as the first choice of drug for the disease ${ }^{[3]}$. However, the long-term intake of Western medicine brings about relatively great toxic and side-effects on the body. Therefore, in clinical practice, a combination of Chinese and Western medicine treatment has also been carried out.

According to traditional Chinese medicine, the category of arrhythmia, appetite loss, and palpitations is caused by imbalance of $q i$ and blood, yin and yang, and dysfunction of the mind, which cause blood stasis to block the heart. In clinical practice, treatment mainly focuses on promoting blood circulation, nourishing yin and nourishing blood. Shensong Yangxin capsule is composed of ginseng, salvia miltiorrhiza, Ophiopogon japonicus, Gansong, dogwood and other Chinese medicinal materials. It has the effects of nourishing yin and rejuvenating the veins, improving the metabolism of myocardial cells, reducing oxygen consumption and oxygen consumption index, playing a role in integral regulation ${ }^{[4]}$. The combined use of Shensong Yangxin capsules and amiodarone can maximize the efficacy of the two, which not only relieves the symptoms but also reduces the harm of side-effects.

In this study, the observation group was given Shensong Yangxin capsules in combination with amiodarone, and it was significantly better than the control group in the comparison of treatment effects, heart rate variability indices, inflammatory factor parameters, and adverse reactions, statistically significant $(\mathrm{P}<0.05)$.

In conclusion, the combined use of Shensong Yangxin capsules and amiodarone has more significant therapeutic effects in the treatment of patients with arrhythmia, which can effectively improve the patient's heart rate variability indices, reduce the inflammatory factors, and relieve the symptoms in patients. The incidence of adverse reactions is low and the safety is quite high, therefore it has great value in clinical applications.

\section{References}

[1] Boos CJ, Holdsworth DA, Woods DR, et al. Assessment of cardiac arrhythmias at extreme high altitude using an implantable cardiac monitor ; REVEAL HA ( REVEAL High Altitude) Study[J].Circulation,2017,135(8): 812-814.

[2] Tian ZY. Clinical observation on the treatment efficacy of Shensong Yangxin capsule in treating arrhythmia of coronary heart disease $[\mathrm{J}]$.Xinxueguanbing Fangzhi Zhishi, 2019, 2 (Mid-term): 47-49.

[3] Liu Q. Clinical observation on amiodarone and Shensong Yangxin capsules in the treatment of patients with arrhythmia $[\mathrm{J}]$. Chinese Journal of Integrative Medicine on Cardio-/Cerebrovascular Disease, 2017, 15(17): 2135-2138.

[4] Hou WQ. Clinical study of Shensong Yangxin capsules combined with amiodarone in the treatment of chronic heart failure and arrhythmia [J]. Chinese Journal of Rational Drug Use, 2018, 15(01):44-46. 